(C) 2006 IEEE. Personal use of this material is permitted. However, permission to reprint/republish this material for advertising or promotional purposes or for creating new collective works for resale or redistribution to servers or lists, or to reuse any copyrighted component of this work in other works must be obtained from the IEEE. 


\title{
A Neural Network based Technique for Automatic Classification of Road Cracks
}

\author{
Justin Bray, Brijesh Verma, Xue Li, and Wade He
}

\begin{abstract}
This paper presents a neural network based technique for the classification of segments of road images into cracks and normal images. The density and histogram features are extracted. The features are passed to a neural network for the classification of images into images with and without cracks. Once images are classified into cracks and non-cracks, they are passed to another neural network for the classification of a crack type after segmentation. Some experiments were conducted and promising results were obtained. The selected results and a comparative analysis are included in this paper.
\end{abstract}

\section{INTRODUCTION}

$\mathrm{T}$ HE aim of this research is to develop a technique that can automatically scan images captured by a road vehicle and detect damage in the road surface. Most current systems for crack detection and classification are costly (in terms of labour), inefficient and unreliable. In most semiautomatic systems, the scanned images are stored on a harddrive and the expert must open the image, detect defect (if any), classify the type of defect, attach a time, date and position, move and store the image and delete any images that do not contain defects. Usually the images are taken every three meters which means that one kilometre of road requires 333 images. Considering there are thousands of kilometres of roads, there is a considerable amount of difficult and challenging work in dangerous working conditions on roads need to be undertaken. Some systems use automatic algorithms for crack detection, however due to irregularities in road surface, variations in road texture, and lighting conditions, and various other difficult environmental conditions, high success in terms of classification rate has not been achieved yet. Therefore, there is a need for more research in investigating and developing new intelligent techniques for crack detection and classification.

This paper is divided into 6 sections. Following this section, Section 2 presents a literature review in the area of crack detection and classification. Section 3 presents the proposed technique. The experimental results are presented

This work was supported in part by the CQU and Canal Industrial and Trading Company.

B. Verma is with Central Queensland University, Australia (phone: +61749309058; fax: +61749309027; e-mail: b.verma@cqu.edu.au).

J. Bray and W. He are with Canal Industrial \& Trading Company, Brisbane, Australia

$\mathrm{X} . \mathrm{Li}$ is with the University of Queensland, Australia. in Section 4. Section 5 presents a discussion of the results. Finally the section 6 concludes the paper.

\section{BACKGROUND}

A review of various methods for the detection and classification of road cracks which have been published in the literature is presented in this section.

Segmentation of the crack from the image is possibly the most difficult procedure in road crack detection. The typical output of this procedure is a binary image where black pixels represent the crack pixels. Thresholding of the image is not simple as there are many variations in road texture and lighting. The human eye is able to perceive even faint cracks when examining the entire image. Thresholding relates to calculating a grey level value in which each pixel is either binarised to 1 or 0 . The difficulty is in choosing this value. Choosing an overall threshold value is difficult which is why the trend has been to break the image into tiles and perform local thresholding on these. This method is chosen in [1-5]. Within each tile the mean grey value is used as a parameter to determine the threshold. In [3] the threshold value is a linear function of the mean grey value. The function was determined statistically by humans and a best fit equation was determined. In [2, 6, 7] a neural network was used to determine the threshold where the inputs were the mean, standard deviation and co-occurrence parameter of the tile. With these segmented images a defect can be determined by the amount of crack pixels in the binary image.

Other methods such as described in [8] determine cracks numerically based on the premise that cracks are both concave and saddle points. In [9] the black pixels are extracted by incrementing a minimum grey level for the entire image and thresholding all pixels above that value. A method described in $[10,11]$ uses a global threshold for the image after it has been normalised. The threshold is calculated with a lookup table style based on mean and standard deviation of the entire image. The data was processed by hand.

Some other methods are used only to determine if an image has a defect for use in real time data collection systems. In [12] the entire image was used as an input to a pulse coded neural network. The output is a barcode in time where the spikes represent brightness and proximity. The barcode is then used as the input into another pulse 
code neural network to determine whether image has a defect of not.

In some thresholding situations pixels are checked for their connectivity or proximity to other pixels. In this way noise from the thresholding can be eliminated. These algorithms occur in $[2,8,13]$.

In [8] the cracks are hypothesises as both concave and saddle points of an image in greyscale. Second derivatives are chosen to select points in the image which are locally concave. Pixels are then given a threshold to convert to binary. Scale of success is not reported.

In some algorithms prior knowledge of the surface texture of sample data from other statistical information is needed which inhibits the development if real images are not available. The human eye can perceive a crack in an image with no prior knowledge or additional information other than that contained within a single image. The method in [13] claim their method has this characteristic.

In [14] the idea of using wavelets to detect cracks is presented. It uses wavelet coefficients to detect and isolate the cracks from the image. Wavelets present an opportunity to use both amplitude and proximity/frequency together which are the characteristics of cracks. They are darker in amplitude and are close together. The images are not adjusted for uniform brightness and it represents one feature that should be included before isolation and detection can be performed.

Thresholding based on means and averages tend rely on data already obtained and matching the results to fit. The Wavelet approach deserves attention because of its ability to perform a type of analysis that performs both magnitude and frequency simultaneously. This is ideal for crack detection where a crack is defined as darker pixels against the background that are in close proximity to each other. The methods of variable thresholding by non-wavelet methods tends to produce various isolated pixels and islands that require further processing to remove. The wavelet function would denote an isolated dark pixel with a low wavelet coefficient and hence becomes filtered automatically. An added benefit is that when the images are saved they are compressed by a ratio of (CR) 50:1 [14] with noise removed. Noise reduction and image compression are simultaneously achieved. Preliminary experiments presented in [14] also show the wavelet transform coefficient calculations to be extremely fast.

Artificial Life (AL) algorithms can also be used in image segmentation [15]. This uses a bottom-up approach which searches for artificial structures within the image. The authors report the method has strong effect on noise removal, oil stain elimination and dark spots. Images are normalised differently by including a bottom-hat algorithm. $\mathrm{AL}$ has been used in image segmentation before but this is the first reported article that uses AL for pavement distress segmentation.

This paper investigates ways to filter defect and no defect images as well as segmentation and type classification afterwards.

\section{PROPOSED TECHNIQUE}

An overview of the proposed technique for crack detection and classification is presented in Fig 1. Two hierarchical neural networks are used, first one for finding defect/no-defect images and second one for the type of defect. Density and histogram features have been extracted from the segmented images and fed to a neural network to exclude/remove images from the database with no cracks (no defects). All images with cracks are passed to another neural network which aims to find the type of defect.

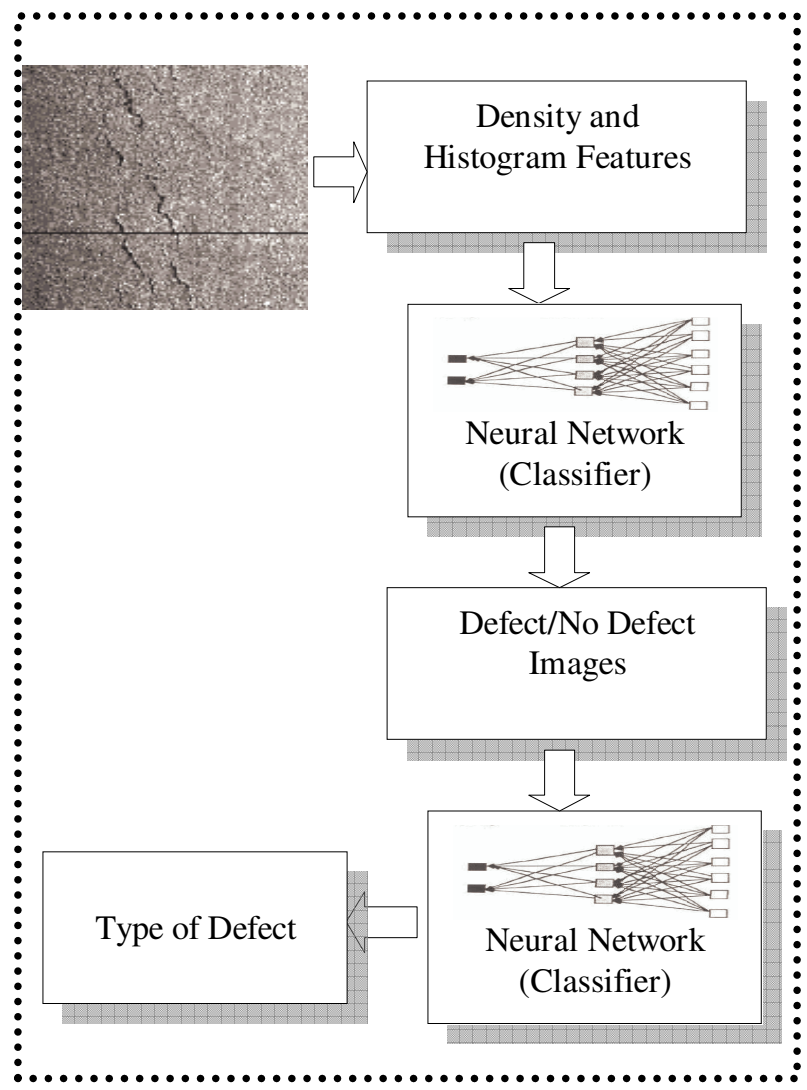

Fig. 1. Proposed Technique.

\section{EXPERIMENTAL RESULTS}

This section deals with the experiments using various existing and proposed methods of segmentation as well as initial tests of the defect/no defect classifier and type of defect classifier.

\section{A. Experiments with a Tile Image Generation}

The first trials were performed on a rough image that includes dark shading. It includes dark cracks and a brightness gradient. This presents a difficult image to 
process. Also an artificial image is generated to perform similar tests on. The first experiments were performed without normalisation of the brightness. The images were processed with a $5 \times 5$ median filter as in [1] to reduce the noise. The resulting images are then processed using variable thresholds for each of the tiles. Each tile was chosen to be $40 \times 40$ pixels as in [1]. This results in the following crack tile images:
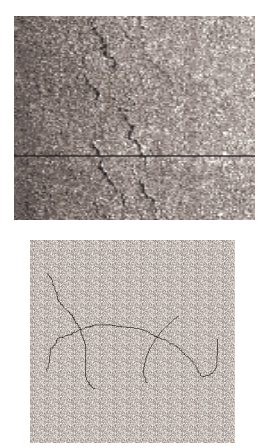
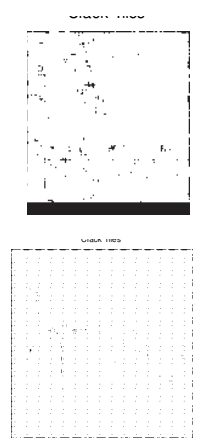

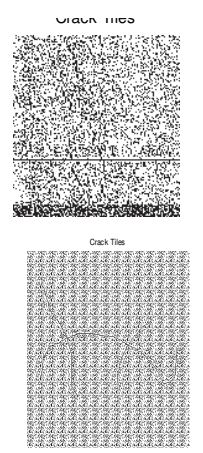

It can be seen that the images are of poor quality and that these affect the way the image thresholding results. It is somewhat effective in that the areas where cracking appears are detected but isolation and other noise produces negative results.

The same thresholding technique is then done again but this time a normalisation is used. This method of normalisation is to blur the image with a $4 \times 4$ average filter to obtain an approximate gradient. Then a scale factor is used.

$$
\operatorname{norm}(i, j)=\operatorname{orig}(i, j) \times k(i, j) k(i, j)=\frac{\text { mean }}{\operatorname{ave}(i, j)}
$$

The mean is the mean grey value of the entire image. The results here are thresholding after the normalisation but without median filtering.

When applying the median filter to the new images the apparent threshold was too high resulting in many of the crack pixels being removed when the standard parameters were used. This indicates that the pictures were not consistent with the pavement images used.

It is therefore important that the images are of good quality with good light conditions to make the job of thresholding using this method effective.

\section{B. Experiments with Wavelets}

Another method of segmentation is to use wavelets. Some experimental results used these images for processing. The Images were analysed with a Daubechies wavelet with an order of 2 . These are then decomposed into three levels $\mathrm{HL}_{\mathrm{k}}, \mathrm{LH}_{\mathrm{k}}$ and $\mathrm{HH}_{\mathrm{k}}$ which relate to the details in the horizontal, vertical and diagonal directions respectively. Three levels of analysis are used. First the normalised image of the road crack is analysed. As can be seen the white pixels represent wavelet coefficients that are large and the dark background represents small wavelet coefficients.
Each row represents the level of analysis for $\mathrm{k}=1,2$ and 3 . The first column is approximations in each level and the second, third and fourth columns represent the horizontal, vertical and diagonal coefficients. The distresses are typically high frequency components and appear as highamplitude wavelet coefficients (WCs). The noise is typically low amplitude WCs in the high frequency sub bands of HL, LH and HH. The background appears in the low frequency sub band of $\mathrm{LL}_{3}$. $\mathrm{LL}_{0}$ is the original image and LL is the approximations as seen in column 1.

The original image can be seen with the same analysis here:

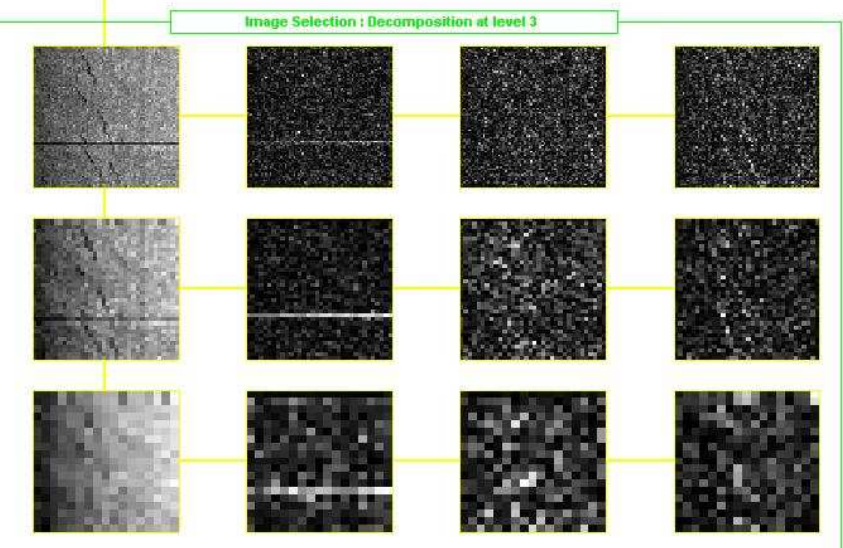

The synthetic image can be seen here:

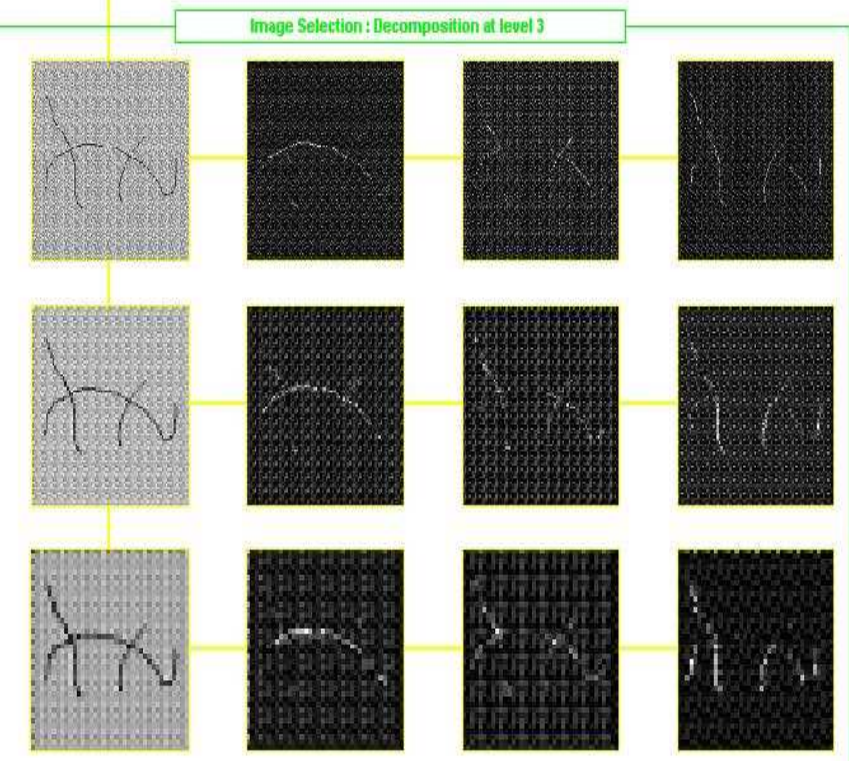

These results here are only the calculation of the coefficients. A thresholding technique is then applied by eliminating the low value coefficients. The pixels are binarised similar to the previous methods by thresholding the coefficients in the high-frequency sub band that are above a predetermined level. This level is determined experimentally but should provide an easier method for 
determining this value. The value of this threshold should be investigated as this could be fixed or a percentage of the maximum level.

Where

$$
D_{1}^{H}=\left\{\begin{array}{l}
1 \text { if } H L_{1}(i, j) \geq C t h \\
0 \text { if } H L_{1}(i, j)<C t h
\end{array}\right.
$$

represents the threshold level for the horizontal direction. This is also done for vertical and diagonal directions resulting in three binary images. These were then normalised to between 0 and 1 . These are then combined to form the complete image with a chosen threshold of 0.75 . Combining the three images using the normalised crack, raw crack and synthetic gives the following results:
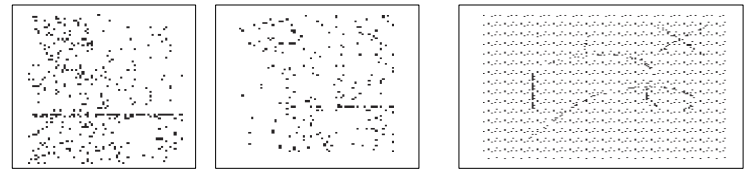

The synthetic image was made of small background tiles. A pure black line was drawn onto the image. Experimental results show that the tile pattern produced periodic glitches that were detected in the wavelet. Their periodic nature caused them to be detected and as such synthetic images may cause problems.

While the true segmentation of the cracks was not achieved to a very high extent, the wavelet tools can be used to detect whether images do or don't have a distress. The authors of [14] suggest a method of computing a Unified Crack Index using the high-amplitude wavelet coefficient percentage (HAWCP) and the high-frequency energy percentage (HFEP) in either singular of combined indices to detect a level of distress. Their claim is $100 \%$ success with the techniques that only detect distress. Classification success was not reported. The HAWCP is the maximum of the sums of the binarised coefficients in each sub band, normalised by the total number of pixels in each sub band for the first level. The HFEP is the defined as the energy of the high-frequency details over the total energy of an image. Both the HAWCP and the HFEP range form 0 to 1 . Another parameter the pavement distress quantification (PDQ) is found by PDQ = HAWCP * HFEP which also ranges from 0 to 1 . Experiments using the PDQ have not been conducted.

\section{Experiments with Neural Networks}

The proposed technique uses neural network as a classifier. In this experiment a neural classifier is trained with a resilient back propagation (RP) algorithm. Each layer is fully connected and uses logarithmic sigmoid transfer function for each layer. Initial experiments using every pixel as an input failed to converge to the desired goal. As such the training data was inconclusive and testing data would prove useless.

The further experiments used the vertical and horizontal densities. This was computed by calculating the average

grey value of a row/column and then tabulating a histogram of these average grey values. The histograms were then normalised by dividing each histogram value by the maximum value of the largest histogram value. The neural network was set to have two outputs, [y1 y2] where [0 1 1] and $\left[\begin{array}{ll}1 & 0\end{array}\right]$ represent no-defect and defect respectively. The classification of the test images output was determined by whether $\mathrm{y} 1>\mathrm{y} 2$ and no thresholds were used. The results are presented below:

There are 25 images with defects and 25 without. Each image is 366 x 300 pixels in size.

Vertical/ horizontal density feature vector:
Learning rate $=1 \mathrm{e}-3$
Min gradient $=0$
Momentum $=1 \mathrm{e}-3$
Inputs $=512(2 \times 256)$
Goal $=0$

TABLE I

RESULTS USING VERTICAL/HORIZONTAL DENSITY FEATURE VECTOR

\begin{tabular}{|c|c|c|c|c|c|}
\hline \multirow{2}{*}{ 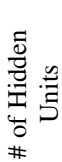 } & \multirow{2}{*}{$\begin{array}{l}\frac{2}{0} \\
\delta \\
\stackrel{0}{0} \\
\text { पै } \\
\#\end{array}$} & \multicolumn{2}{|c|}{$\begin{array}{c}\text { Training Set } \\
\text { Classification Rate } \\
{[\%]}\end{array}$} & \multicolumn{2}{|c|}{$\begin{array}{c}\text { Test Set } \\
\text { Classification Rate } \\
{[\%]}\end{array}$} \\
\hline & & Defect & $\begin{array}{c}\text { No } \\
\text { Defect }\end{array}$ & Defect & $\begin{array}{c}\text { No } \\
\text { Defect }\end{array}$ \\
\hline 6 & 100 & 100 & 100 & 78 & 74 \\
\hline 6 & 1000 & 100 & 100 & 68 & 60 \\
\hline 12 & 100 & 100 & 100 & 68 & 70 \\
\hline 12 & 1000 & 100 & 100 & 76 & 70 \\
\hline 24 & 100 & 100 & 100 & 66 & 78 \\
\hline 24 & 1000 & 100 & 100 & 48 & 78 \\
\hline 36 & 100 & 100 & 100 & 56 & 76 \\
\hline 36 & 1000 & 100 & 100 & 38 & 84 \\
\hline
\end{tabular}

Histogram feature vector:
Learning rate $=1 \mathrm{e}-2$
Momentum $=1 \mathrm{e}-2$
Goal $=0$
Min gradient $=0$
Inputs $=256$

TABLE II

RESULTS USING HISTOGRAM FEATURE VECTOR

\begin{tabular}{|c|c|c|c|c|c|}
\hline \multirow{2}{*}{ 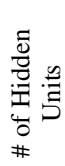 } & \multirow{2}{*}{ 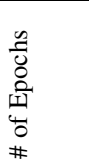 } & \multicolumn{2}{|c|}{$\begin{array}{c}\text { Training Set } \\
\text { Classification Rate } \\
{[\%]}\end{array}$} & \multicolumn{2}{|c|}{$\begin{array}{c}\text { Test Set } \\
\text { Classification Rate } \\
{[\%]}\end{array}$} \\
\hline & & Defect & $\begin{array}{c}\text { No } \\
\text { Defect }\end{array}$ & Defect & $\begin{array}{c}\text { No } \\
\text { Defect }\end{array}$ \\
\hline 6 & 1000 & 100 & 100 & 100 & 82 \\
\hline 6 & 10000 & 100 & 100 & 100 & 76 \\
\hline 12 & 1000 & 100 & 100 & 100 & 80 \\
\hline 12 & 10000 & 100 & 100 & 100 & 76 \\
\hline 24 & 1000 & 100 & 100 & 100 & 80 \\
\hline 24 & 10000 & 100 & 100 & 100 & 80 \\
\hline 36 & 1000 & 100 & 100 & 100 & 80 \\
\hline 36 & 10000 & 100 & 100 & 100 & 80 \\
\hline
\end{tabular}

Subsequent tests were performed using the histogram input features to classify cracks in to alligator, block, vertical and horizontal types into their respective outputs [y1 y2 y3 y4]. For example, $\left[\begin{array}{llll}0 & 1 & 0 & 0\end{array}\right]$ would represent block type. The output is classified by the $\max (\mathrm{y} 1, \mathrm{y} 2, \mathrm{y} 3, \mathrm{y} 4)$. Ten images of each type of defect were used for training and testing. 
Histogram feature vector:

$$
\begin{array}{ll}
\text { Learning rate }=1 \mathrm{e}-2 & \text { Min gradient }=0 \\
\text { Momentum }=1 \mathrm{e}-2 & \text { Inputs }=256 \\
\text { Goal }=0 &
\end{array}
$$

\begin{tabular}{|c|c|c|c|c|c|c|c|c|c|}
\hline \multirow{2}{*}{ 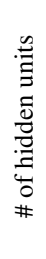 } & \multirow{2}{*}{ 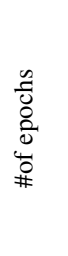 } & \multicolumn{4}{|c|}{$\begin{array}{c}\text { Training Set } \\
\text { Classification Rate [\%] }\end{array}$} & \multicolumn{4}{|c|}{$\begin{array}{c}\text { Test Set } \\
\text { Classification Rate [\%] }\end{array}$} \\
\hline & & 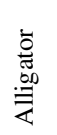 & $\frac{\frac{u}{0}}{\infty}$ & 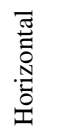 & $\begin{array}{l}\overline{0} \\
\stackrel{0}{0} \\
> \\
>\end{array}$ & 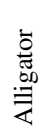 & $\frac{\ddot{0}}{0}$ & 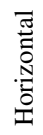 & $\begin{array}{l}\stackrel{\widetilde{D}}{\underbrace{}_{0}} \\
>\end{array}$ \\
\hline 6 & $10 \mathrm{e} 5$ & 100 & 80 & 70 & 80 & 40 & 20 & 70 & 50 \\
\hline 6 & $10 \mathrm{e} 6$ & 100 & 100 & 70 & 70 & 30 & 50 & 30 & 10 \\
\hline 12 & $10 \mathrm{e} 5$ & 100 & 100 & 100 & 90 & 40 & 50 & 60 & 10 \\
\hline 12 & $10 \mathrm{e} 6$ & 100 & 100 & 100 & 90 & 30 & 50 & 60 & 20 \\
\hline 24 & $10 \mathrm{e} 5$ & 100 & 90 & 100 & 90 & 40 & 30 & 80 & 20 \\
\hline 24 & $10 \mathrm{e} 6$ & 100 & 80 & 90 & 100 & 40 & 60 & 50 & 10 \\
\hline 36 & $10 \mathrm{e} 5$ & 100 & 80 & 100 & 90 & 30 & 60 & 70 & 30 \\
\hline 36 & $10 \mathrm{e} 6$ & 100 & 100 & 100 & 100 & 30 & 50 & 20 & 60 \\
\hline
\end{tabular}

TABLE III

RESULTS USING HISTOGRAM FEATURE VECTOR

\section{Discussion AND ANALYSIS OF RESUlts}

The results of the local thresholding technique on tile images show promise but with the images used the method showed its limitation. Lots of isolated pixels were generated. The lighting conditions also influenced the results. This method alone achieved somewhat reasonable results but would not be considered good.

The wavelet methods presented are not well documented in the literature on crack detection. This method alone is below average but shows promise in the detection of the peak cracks. It could be used to determine the local thresholds and combined with the tile thresholding technique. The artificial image generated noise in the image due to the repetitive nature of the image. Although true images may not produce these results it is interesting to note.

The proposed method performed well with artificial images. The results of the vertical and horizontal density input achieved around $100 \%$ classification rate. The detection of images that had defect was higher overall. This may be attributed to the common background that was used. The images that were detected incorrectly were consistent over the range of hidden units. These were images with a lighter background or the method used to generate the artificial images. Overall this method is promising and further analysis is required when real images are obtained.

The results of using the histogram of defect images as input to the neural network for the defect type classification produced poor results. The defect type classification results are being analysed for finding cause of poor performance.

\section{CONCLUSIONS}

We have presented a neural network based technique to classify images with cracks and without cracks.
Segmentation presents a difficult problem to the detection of cracks. As seen by the results a single statistical method on its own is not capable of producing a robust repetitive result. Possible solutions are to combine these methods to produce a means of isolating crack pixels. A wavelet based approach can detect the peaks in the images which represent the cracks. While noise is still apparent this could be combined with the local thresholding in the vicinity of the peaks and repeated.

The proposed neural network based technique has been tested with various artificial images with cracks and nocracks. Some promising results such as $100 \%$ classification rate (images with cracks) and $82 \%$ classification rate (images without cracks) have been obtained. The results confirm that the proposed approach is an appropriate tool for crack detection. However, the approach didn't perform well on defect type classification. Poor results were obtained with the histogram input for the classification of defect type.

In our future work, more experiments will be conducted and the second part (defect type classification) of the proposed approach will be tested by incorporating and combining feature extraction techniques other than histogram which did not perform well. The experiments with neural networks in conjunction with wavelet and artificial life techniques were not conducted as yet. Their true success at filtering and segmentation techniques over a broad range of images will be of interest as very little published literature exists within these fields.

\section{REFERENCES}

[1] S. Jitprasithsiri, H. Lee, R. G. Sorcic, and R. Johnston, "Development of digital imageprocessing algorithm to compute unified crack index for Salt Lake City," Transportation Research Record, vol. 1526, pp. 142-148, 1996.

[2] M. S. Kaseko and S. G. Ritchie, "Neural network based methodology for pavement crack detection and classification," Transportation Research Part C: Emerging Technologies L2, vol. 1, pp. 275, 1993.

[3] B. J. Lee and H. D. Lee, "Position-invariant neural network for digital pavement crack analysis," Computer-Aided Civil and Infrastructure Engineering, vol. 19, pp. 105-118, 2004.

[4] H. N. Koutsopoulos and A. B. Downey, "Primitivebased classification of pavement cracking images," Journal of Transportation Engineering, vol. 119, pp. 402-418, 1993.

[5] H. N. Koutsopoulos, V. I. Kapotis, and A. B. Downey, "Improved methods for classification of pavement distress images," Transportation Research, vol. 2, pp. 19-33, 1994.

[6] M. S. Kaseko, Z.-P. Lo, and S. G. Ritchie, "Comparison of traditional and neural classifiers for pavement-crack detection," Journal of 
Transportation Engineering, vol. 120, pp. 552-569, 1994.

[7] M. Kaseko, S., S. G. Ritchie, and Z.-P. Lo, "Evaluation of two automated thresholding techniques for pavement images," presented at Proceedings of the Infrastructure Planning and Management, Jun 21-23 1993, Denver, CO, USA, 1993.

[8] M. D. Ginsberg and M. Y. Shahin, "Algorithm for crack detection in automated pavement analysis," presented at Proceedings of the Infrastructure Planning and Management,, Denver, CO, USA, 1993.

[9] N. Tanaka and K. Uematsu, "A crack detection method in road surface images using morphology," presented at IAPR Workshop on Machine vision Applications, Makuhari, Chida, Japan, 1998.

[10] H. D. Cheng, X. J. Shi, and C. Glazier, "Real-time image thresholding based on sample space reduction and interpolation approach," Journal of Computing in Civil Engineering, vol. 17, pp. 264272, 2003.

[11] H. D. Cheng, J. Wang, Y. G. Hu, C. Glazier, X. J. Shi, and X. W. Chen, "Novel approach to pavement cracking detection based on neural network," Transportation Research Record, pp. 119-127, 2001.

[12] H. C. S. Rughooputh, S. D. D. V. Rughooputh, and J. Kinser, "Automatic Inspection of road surfaces," Proceedings of SPIE - The International Society for Optical Engineering, vol. 3966, pp. 349-356, 2000.

[13] H. D. Cheng, J.-R. Chen, C. Glazier, and Y. G. Hu., "Novel Approach to pavement cracking detection based on fuzzy set theory," Journal of Computing in Civil Engineering, vol. 13, pp. 270280, 1999.

[14] J. Zhou, P. S. Huang, and F. Chiang, "Wavelet based pavement distress detection and evaluation," Proceedings of the SPIE - The International Society for Optical Engineering Wavelets: application in Signal and Image Processing X, 4-8 Aug. 2003, vol. 5207, pp. 728-39, 2003.

[15] H. G. Zhang and Q. Wang, "Use of Artificial Life System for pavement distress survey," in The 30th Annual Conference of the IEEE Industrial Electronics Society. Busan, Korea, 2004, pp. 24862490. 